\title{
A Pilot Symbol-Aided Technique Used for Digital Signals in Multipath Environments
}

\author{
H. K. Lau and S. W. Cheung \\ Communications Research Group \\ Department of Electrical and Electronic Engineering \\ The University of Hong Kong \\ Pokfulam Road \\ Hong Kong
}

\begin{abstract}
The paper proposes a pilot symbol-aided technique to reduce the effects of multipath fading on digital signals in mobile radio environments. A pilot symbol from a known sequence is multiplexed into the data symbols at regular intervals to form frames of symbols for transmission. Using the pilot symbols in two consecutive frames, the receiver is able to estimate and correct the fading distortion. The proposed technique uses a linear extrapolation approach that requires no storage of previous data symbols and introduces virtually zero delay to the received message. A series of computer-simulation tests has been carried out to assess the performance of the proposed technique on different signal constellations, including the 8PSK, 16PSK, 16QAM and star-16QAM signals, in the Rayleigh fading channels. The results have shown that significant improvements in bit-error rate performances of the systems can be achieved by the proposed technique.
\end{abstract}

\section{Introduction}

In the transmission of digital signals in a mobile radio environment, a serious problem encountered is the fading distortion introduced into the signals by the mobile channel. For a coherent receiver, this will result in losing lock of the phaselocked loop. The normal approach to the problem is to use differential (non-coherent) detection at the receiver, such that a correct carrier phase is not required for data detection.

In the past years, considerable work has been carried out on using different techniques to improve the performances of digital signals in multipath environments. Recently, the pilot tone- and the pilot symbol-aided transmission schemes have been proposed and attracted much attentions [1-6]. In a pilot tone-aided system, the receiver is provided with an explicit amplitude and phase reference through a pilot tone signal for data detection [1-4] While in a pilot symbol-aided system, the data-symbol sequence is divided into frames of symbols for transmission. A known pilot symbol is inserted at the beginning of each frame. At the receiver, these pilot symbols are used for the fading compensation. However, in previous studies [5-6], the receivers are required to store up data symbols before fading correction is applied. As a result, a significant delay may be introduced to the received message by the correction process.
The paper first proposes a simplified pilot symbol-aided technique that uses a linear extrapolation approach and introduces zero delay to the received message. The algorithm only requires two pilot symbols (one in the present frame and the other in the previous frame) to estimate the fading distortion and makes the appropriate correction to the faded signal in the following frame. The implementation of this algorithm is straight forward and requires no storage for the data symbols. The performance of the algorithm in a Gaussian channel is also analyzed in this paper. A series of computer-simulation tests has been carried out to assess the performance of the proposed technique on different signal constellations, including the 8PSK, 16PSK, 16QAM and star-16QAM [7] signals. The results have shown that the proposed technique can significantly improve the bit-error rate performances of these systems, relative to those obtained by differential detection.

\section{Model of System}

The baseband equivalent model of the data-transmission system considered in the study is shown in Fig. 1.

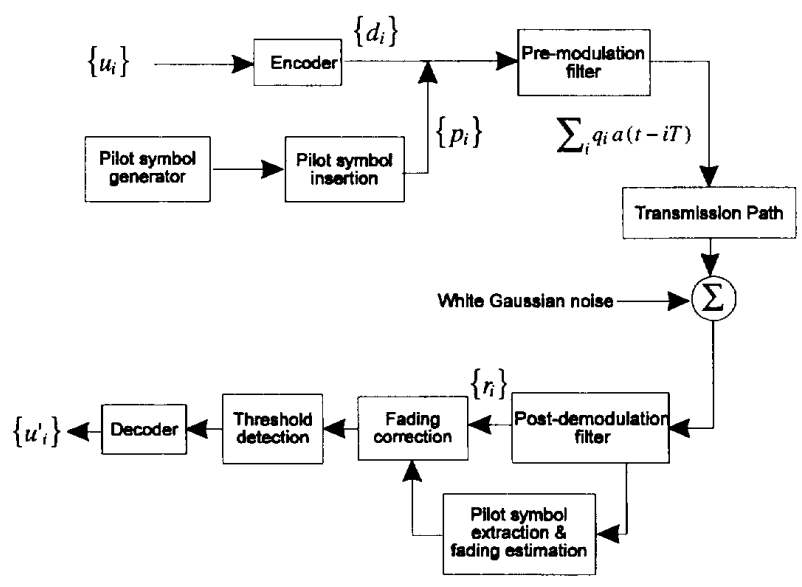

Figure 1 Model of System

The information to be transmitted is carried by the binary digits $\left\{u_{i}\right\}$. When the encoder has received $u_{i}, u_{i+1}, \ldots, u_{i+M-1}$ at time $t=i T$ seconds, it maps these signals into the appropriate 
M-ary data symbols $d_{i}$ according to one of the constellations shown in Fig. 2 . For every $(L-1)$-data symbols, a pilot symbol from $\left\{p_{i}\right\}$ is inserted to form an $L$-symbol frame. The pilot symbols, $\left\{p_{i}\right\}$, are pseudo random and each symbol has the same possible values as the $\left\{u_{i}\right\}$. The receiver, of course, has the prior knowledge of $\left\{p_{i}\right\}$, and so it is able to estimate the fading distortion introduced in the transmission path and makes the appropriate correction to the received data signal. The proposed algorithm for the multipath fading compensation is described in section 3. At time $t=i T$, the data signal symbol is used to form the impulse $q_{i} \delta(t-i T)$, where $q_{i}$ is either a data symbol or a pilot symbol, which is fed to the pre-modulation filter with an impulse response $a(t)$. The signal at the output of the filter is $\Sigma_{i} q_{i} a(t-i T)$ which will linearly modulated, up-converted to $900 \mathrm{MHz}$ and fed to an antenna for transmission.

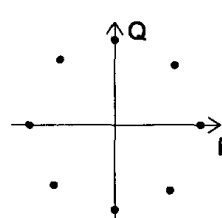

(a)

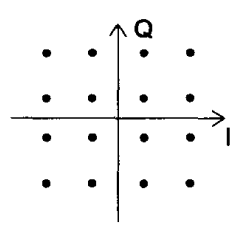

(c)

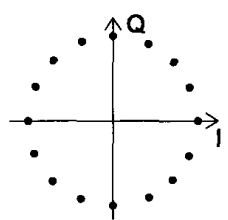

(b)

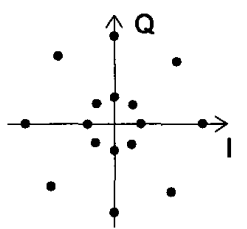

(d)
Figure 2 Signal Constellations. (a) 8PSK, (b) 16PSK, (c) Square-16QAM and (d) Star-16QAM

The transmission path in Fig. 1 is a time-varying linear baseband channel that introduces Rayleigh fading to the transmitted signal and is modeled as in Fig. 3 [8].

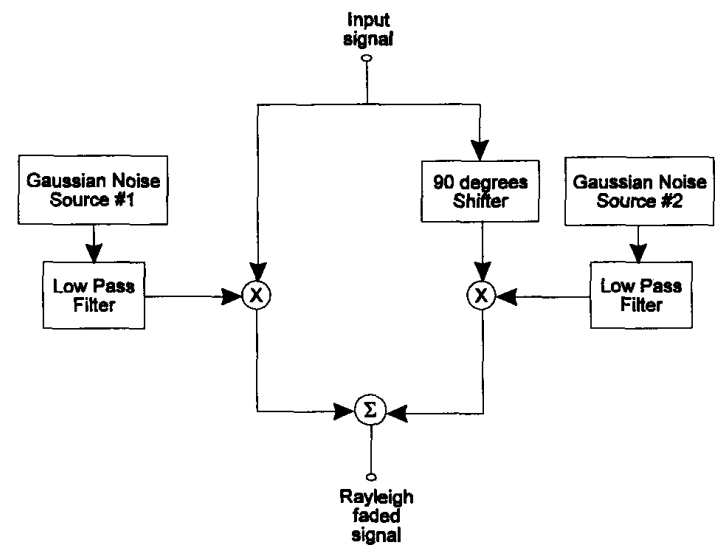

Figure 3 Model of Rayleigh Fading
The lowpass filters shown in Fig. 3 have the normalized characteristic of

$$
\xi(f)= \begin{cases}\frac{1}{\pi \sqrt{f_{d}^{2}-f^{2}}} & \text { when }-f_{d} \leq f \leq f_{d} \\ 0 & \text { elsewhere }\end{cases}
$$

The quantity $f_{d}$ determines the maximum Doppler spread of the faded signal and is given by

$$
f_{d}=\frac{v}{\lambda}
$$

where $v$ is the traveling speed of the vehicle and $\lambda$ is the wavelength of the transmitted carrier frequency.

In Fig. 3, the input signal is multiplied by a complex-valued signal $y(t)$ to give the output faded signal $\sum_{i} q_{i} a(t-i T) y(t)$. Stationary additive white Gaussian noise is assumed to be added at the input of the receiver.

At the receiver, the $900 \mathrm{MHz}$ signal from the antenna is down-converted, demodulated and then filtered by a postdemodulation filter. The latter is taken to have the same impulse response $a(t)$ as the pre-modulation filter to give the corresponding baseband signal

$$
r(t)=\left[\sum_{i} q_{i} a(t-i T) y(t)+w(t)\right] * a(t)
$$

where $*$ denotes the convolution process, $y(t)$ represents the distortion introduced into the data signal by the transmission path, and $w(t)$ is the additive white Gaussian noise with onesided power spectral density of $N_{0}$. Both $y(t)$ and $w(t)$ are complex valued.

If the fading rate is slow compared to the symbol rate, the intersymbol interference (ISI) caused by the filtering process can be neglected. The baseband signal $r(t)$ is sampled at the time instants $\{i T\}$. For convenience, assume $a(t) * a(t)=1$ at time $t=0$. The sample signal at time $t=i T$ is

$$
r_{i}=q_{i} y_{i}+w_{i}
$$

where $y_{i}=y(i T)$ and $w_{i}=w(i T)$.

Assume that frame synchronism has been achieved. Since the sequence of the pilot symbols is known at the receiver, $y_{i}$ can be estimated from the pilot symbols and used to correct the changes in the received data symbols. The threshold detector (Fig. 1) then operates on the resultant data symbols to produce the binary data $\left\{u_{i}^{\prime}\right\}$ at the output.

\section{Pilot Symbol-Aided Technique}

Ideal symbol- and frame-synchronization are assumed here. The position of the pilot symbol within the frame has an insignificant effect on bit-error rate performance [5]. For simplicity, the pilot symbol is assumed to be inserted at the beginning of each frame to form $L$-symbol frames as shown in Fig. 4. 


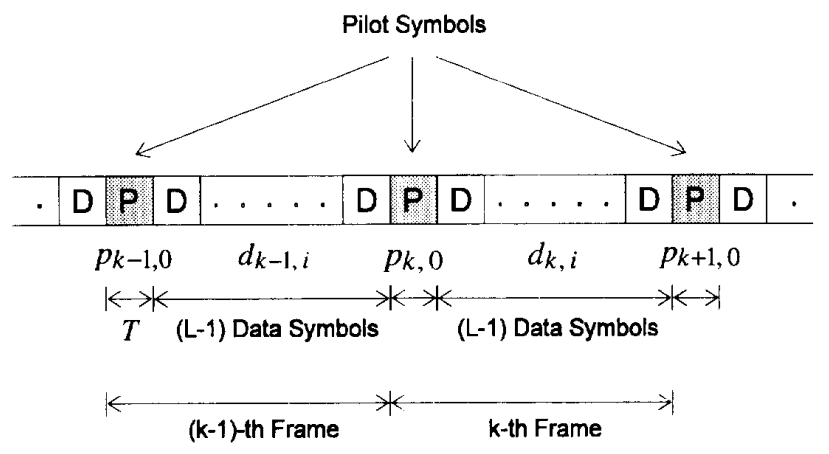

Figure 4 Frame Structure of Transmitted Symbol Sequence

The sample signal in the $i$-th position of the $k$-th frame can be written as (from Eqn. 4)

$$
\eta_{k, i}=q_{k, i} y_{k, i}+w_{k, i}
$$

where $q_{k, i}$ is either a pilot symbol or a data symbol.

For a pilot symbol, $i=0$, and the sample signal is

$$
r_{k, 0}=p_{k, 0} y_{k, 0}+w_{k, 0}
$$

where $p_{k, 0}$ is the pilot symbol in the $k$-th frame.

For a data symbol, $i=1,2, \ldots, L-1$, and the sample signal is

$$
r_{k, i}=d_{k, i} y_{k, i}+w_{k, i}
$$

where $d_{k, i}$ are the data symbols in the $k$-th frame.

In Eqn. 6, $p_{k, 0}$ is known at the receiver. At high signal-tonoise ratios (i.e., $p_{k, 0} y_{k, 0} \gg w_{k, 0}$ and $p_{k-1,0} y_{k-1,0} \gg w_{k-1,0}$ ), good estimates of $y_{k, 0}$ and $y_{k-1,0}$ in the present and previous pilot symbols can be obtained as

$$
\hat{y}_{k, 0}=\frac{\eta_{k, 0}}{p_{k, 0}} \text { and } \hat{y}_{k-1,0}=\frac{\eta_{k-1,0}}{p_{k-1,0}}
$$

If the fading process is sufficiently slow, linear extrapolation technique can be used to estimate $y_{k, 1}$ to $y_{k, L-1}$ from $\hat{y}_{k, 0}$ and $\hat{y}_{k-1,0}$ using the relationship

$$
\hat{y}_{k, i}=\left(1+\frac{i}{L}\right) \hat{y}_{k, 0}-\left(\frac{i}{L}\right) \hat{y}_{k-1,0}
$$

for $i=1,2, \ldots, L-1$.

The faded signal sample $r_{k, i}$ (Eqn. 7) in $i$-th position of the $k$-th frame can then be corrected to produce an estimate of the data symbol

$$
\hat{r}_{k, i}=\frac{r_{k, i}-w_{k, i}}{\hat{y}_{k, i}} \approx \frac{\eta_{k, i}}{\hat{y}_{k, i}}
$$

Due to the noise terms $w_{k, 0}$ and $w_{k-1,0}$, perfect estimation is not possible. However, in a slowly faded channel and at high signal-to-noise ratios $\left(r_{k, 0}>w_{k, 0}\right.$ and $\left.r_{k-1,0}>w_{k-1,0}\right)$, good estimates of the changes introduced in the transmission path can still be obtained.
One of the main advantages of the proposed algorithm is that it uses a linear extrapolation and so introduces no delay to the received message. However, since the pilot symbols carry no data information, the information rate of the system is reduced. To maintain the same throughput of the system, the data rate must be increased by a factor of $L /(L-1)$. In addition, since a portion of the transmitted signal power is used for transmitting the pilot symbols, for a given transmitted power of the system, the energy per data symbol is reduced by factor of $10 \times \log [L /(L-1)] \mathrm{dB}$.

\section{Degradation in Gaussian Channel}

From Eqns. 8 and 6, Eqn. 9 can be written as

$\hat{y}_{k, i}=\left(y_{k, 0}+\frac{w_{k, 0}}{p_{k, 0}}\right)+\left(\frac{i}{L}\right)\left(y_{k, 0}-y_{k-1,0}+\frac{w_{k, 0}}{p_{k, 0}}-\frac{w_{k-1,0}}{p_{k-1,0}}\right)$

In a Gaussian channel, $y_{k, i}=1$ for all $k$ and $i$, thus the estimate of $y_{k, i}$ is

$$
\hat{y}_{k, i}=\left(1+\frac{w_{k, 0}}{p_{k, 0}}\right)+\left(\frac{i}{L}\right)\left(\frac{w_{k, 0}}{p_{k, 0}}-\frac{w_{k-1,0}}{p_{k-1,0}}\right)
$$

To simplify the analysis, assume the same symbol is used for all the pilot symbol transmission, i.e., $p_{k, 0}=p_{k-1,0}=p_{k}$, Eqn. 12 becomes

$$
\hat{y}_{k, i}=1+\frac{1}{p_{k}}\left[\left(1+\frac{i}{L}\right) w_{k, 0}-\left(\frac{i}{L}\right) w_{k-1,0}\right]
$$

where $w_{k, 0}$ and $w_{k-1,0}$ have zero mean and fixed variance of $\sigma^{2}$.

The variance of $\hat{y}_{k, i}$ is given by

$$
\sigma_{\hat{y}}^{2}=\frac{\sigma^{2}}{\left|p_{k}\right|^{2}(L-1)} \sum_{i=1}^{L-1}\left[1+2\left(\frac{i}{L}\right)+2\left(\frac{i}{L}\right)^{2}\right]
$$

From Eqn. 7, with $y_{k, i}=1$, the received data signal is

$$
r_{k, i}=d_{k, i}+w_{k, i}
$$

Substituting Eqns. 13 and 15 into Eqn. 10, the received data symbol after fading correction is

$$
\hat{\eta}_{k, i}=\frac{d_{k, i}+w_{k, i}}{1+y(k, i)}
$$

where $y(k, i)=\frac{1}{p_{k}}\left[\left(1+\frac{i}{L}\right) w_{k, 0}-\left(\frac{i}{L}\right) w_{k-1,0}\right]$

If $\left|w_{k, i}\right|^{2}<1$, then Eqn. 16 can be approximated by a Taylor series

$$
\hat{r}_{k, i} \approx d_{k, i}+w(k, i)
$$

where $w(k, i)=w_{k, i}-d_{k, i} y(k, i)$ and has the variance given by

$$
\sigma_{w}^{2}=\sigma^{2}+\sigma_{d}^{2} \sigma_{\hat{y}}^{2}
$$

with $\sigma_{d}{ }^{2}$ the average signal power per symbol. 
Thus, in a Gaussian channel, the proposed pilot symbolaided scheme causes the system a degradation in performance of

$$
10 \log \left(1+\frac{\sigma_{d}^{2} \sigma_{\hat{y}}^{2}}{\sigma^{2}}\right) \mathrm{dB}
$$

\section{Simulation Results and Discussions}

\subsection{Basic Assumptions}

A series of computer-simulation tests has been carried out to evaluate the performance of the system shown in Fig. 1, using the pilot symbol-aided technique described in section 3. Different types of signals, including the 8PSK, 16PSK, 16QAM and star16QAM signals with the signal constellations shown in Fig. 2, have been investigated. The transmission rates used are $24 \mathrm{~kb} / \mathrm{s}$ for $8 \mathrm{PSK}$ and $32 \mathrm{~kb} / \mathrm{s}$ for the rest of the signals. The vehicle is assumed to be traveling at the speeds of $15 \mathrm{~km} / \mathrm{h}$ and $100 \mathrm{~km} / \mathrm{h}$, and the transmitter is operating at $900 \mathrm{MHz}$, leading to the Doppler spreads $\left(f_{d} T\right)$ of about $1.5 \times 10^{-3}$ and $1.0 \times 10^{-2}$ of the symbol rate respectively.

In all tests, baseband equivalent models of the systems have been used. The pre-modulation filter in the transmitter and the post-demodulation filter in the receiver are the same. The resultant transfer function of the filters in cascade has a sinusoidal rolloff of $100 \%$. The signal-to-noise ratio is taken as

$$
S N R=10\left[\log \left(\frac{E_{b}}{N_{0}}\right)\right] \mathrm{dB}
$$

where $E_{b}$ is the average transmitted energy per bit at the output of the transmitter of Fig. 1 and $N_{0}$ is the one-sided power spectral density of the additive white Gaussian noise measured at the same point.

\subsection{Discussion of Results}

The bit-error rate performances of the 8PSK, 16PSK, 16QAM and star-16QAM signals are shown in Figs. 5-8. The pilot symbol-aided technique described in section 3 , with $L=4$, 8 and 16, has been employed in the receivers to correct the faded signals. The performances of the systems using differential detection and coherent detection (i.e., assuming perfect-carrier recovery) are also shown in the corresponding figures for comparison. It is evident that coherent detection is not suitable in Rayleigh fading environments for all these signals.

When the proposed technique is used, these results show that the smaller the value of $L$, the better are the performances of the systems, as might be expected. This is particular true in fast fading conditions because the estimates of the changes in the signals needed to be updated more often. With the vehicle traveling at $15 \mathrm{~km} / \mathrm{h}$, the pilot symbol-aided technique is inferior to the differential detection at low signal-to-noise ratios. This is because the estimates of the changes are unlikely to be very accurate at low signal-to-noise ratios. Under these conditions, the proposed technique is not recommended.
With $L=4$ (i.e., the estimate of $y_{i}$ is updated every three data symbol), for 8PSK and star-16QAM, the technique begins to gain advantages on performance over differential detection at error rates below about $10^{-4}$. It also can be seen that there is no much difference in performances between $L=4$ and $L=8$ at the error rates tested.

When the vehicle is traveling at a higher speed of $100 \mathrm{~km} / \mathrm{h}$, these results show that the performances of all the signals tested become worse. With $L=8$, the technique cannot give better performances than those with differential detection. However, if $L$ is reduced to 4 and at high signal-to-noise ratios, substantial improvements in performances (about one order of magnitude) can be obtained by employing the proposed technique. This is due to the fact that fading is now changing more rapidly and so the estimates of the changes in the signals should be applied to the data signals more often if better performances are to be achieved.

The comparison on the performances of the 16PSK, 16QAM and star-16QAM signals, with $L=4$ in Figs. 6-8 indicate that all signals have about the same performance, with star-16QAM being slightly better than the other two.

\section{Conclusions}

A pilot symbol-aided scheme used for reducing the effects of multipath fading on digital signals in mobile radio environments has been analyzed. The scheme uses a simple linear extrapolation technique that requires no storage of data symbols and introduces virtually zero delay to the received message. The effects of the scheme on the performance of 8PSK, 16PSK, 16QAM and star-16QAM have been investigated by means of computer simulation. Simulation results have shown that, at high signal-to-noise ratios, significant improvements in the bit-error rate performances of the systems can be obtained by the proposed technique, relative to those obtained using differential detection.

\section{References}

[1] J. P. McGeehan and A. J. Bateman, "Phase Locked Transparent Tone-inBand (TTIB): A New Spectrum Configuration Particularly Suited to the Transmission of Data Over SSB Mobile Radio Networks," IEEE Transactions on Communications, vol. 32, pp. 81-87, January 1984.

[2] F. Davarian, "Mobile Digital Communications via Tone Calibration," IEEE Transactions on Vehicular Technology, vol. 36, no. 2, pp. 55-62, May 1987.

[3] M. L. Moher and J. H. Lodge, "TCMP-A Modulation and Coding Strategy for Rician Fading Channel," IEEE Journal on Selected Areas in Communications, vol. 7, pp. 1347-1335, December 1989.

[4] H. W. H. Li and J. K. Cavers, "An Adaptive Filtering Technique for PilotAided Transmission Systems," IEEE Transactions on Vehicular Technology, vol. 40, no. 3, pp. 532-545, August 1991.

[5] J. K. Cavers, "An Analysis of Pilot Symbol Assisted Modulation for Rayleigh Fading Channels," IEEE Transactions on Vehicular Technology, vol. 40, no. 4, pp. 686-693, November 1991.

[6] S. Sampei and T. Sunaga, "Rayleigh Fading Compensation for QAM in Land Mobile Radio Communications," IEEE Transactions on Vehicular Technology, vol. 42, no. 2, pp. 137-147, May 1993.

[7] W. T. Webb, "Modulation Methods for PCNs," IEEE Communications Magazine, vol. 30, no. 12, pp. 90-95, December 1992.

[8] W. C. Y. Lee, Mobile Communications Engineering. New York: McGrawHill, 1983. 


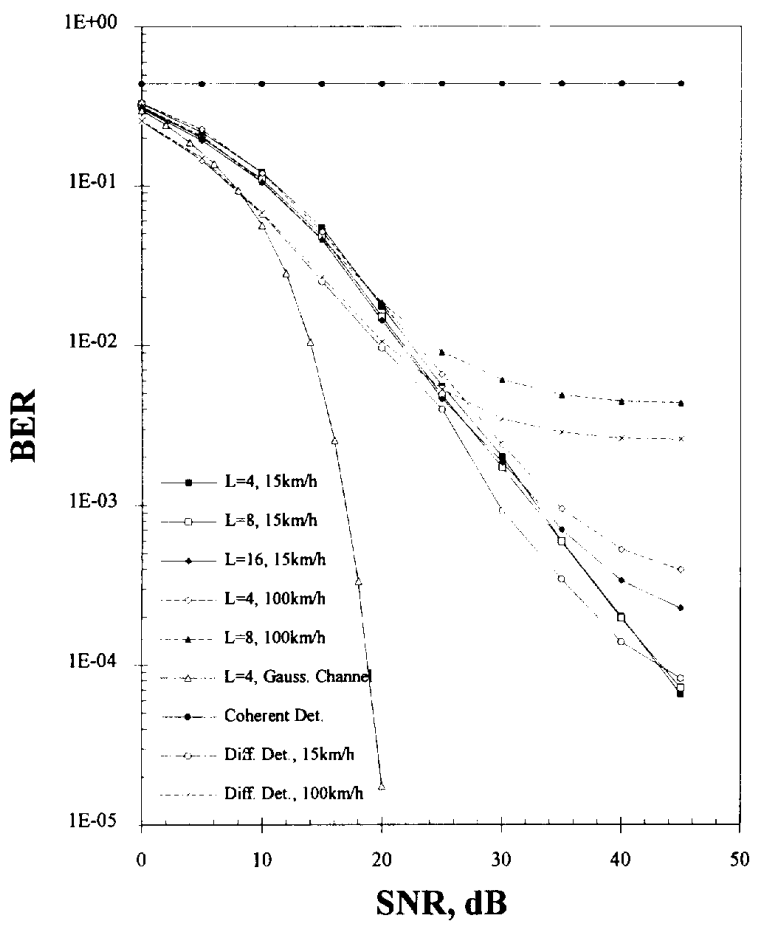

Figure 5 Performance of Pilot Symbol-Aided $8 P S K$ in Rayleigh Channels

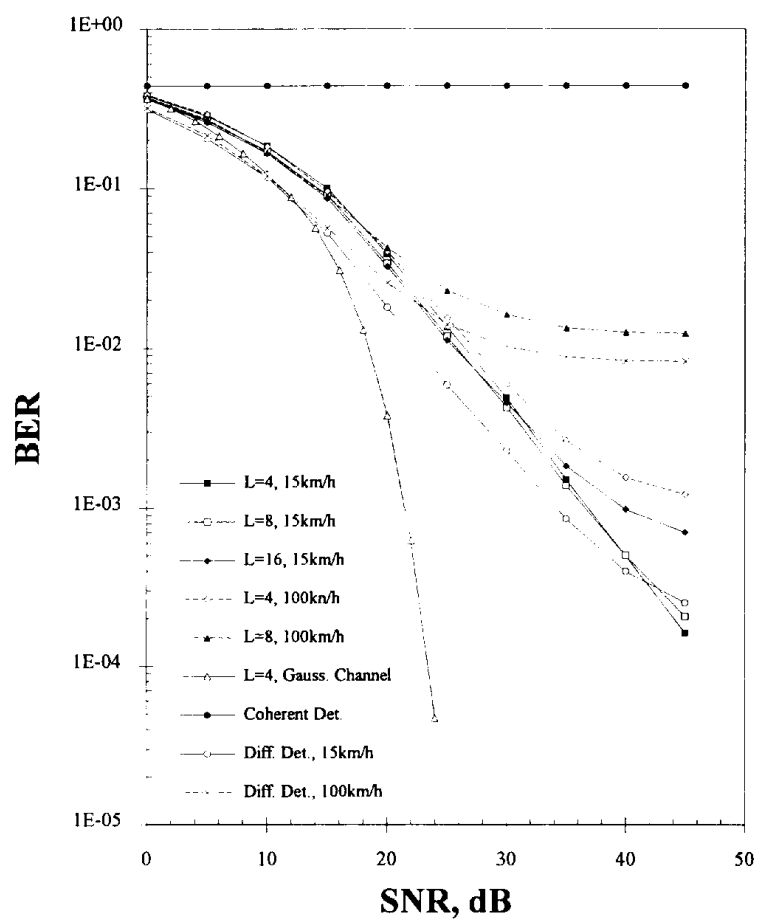

Figure 6 Performance of Pilot Symbol-Aided I6PSK in Rayleigh Channels

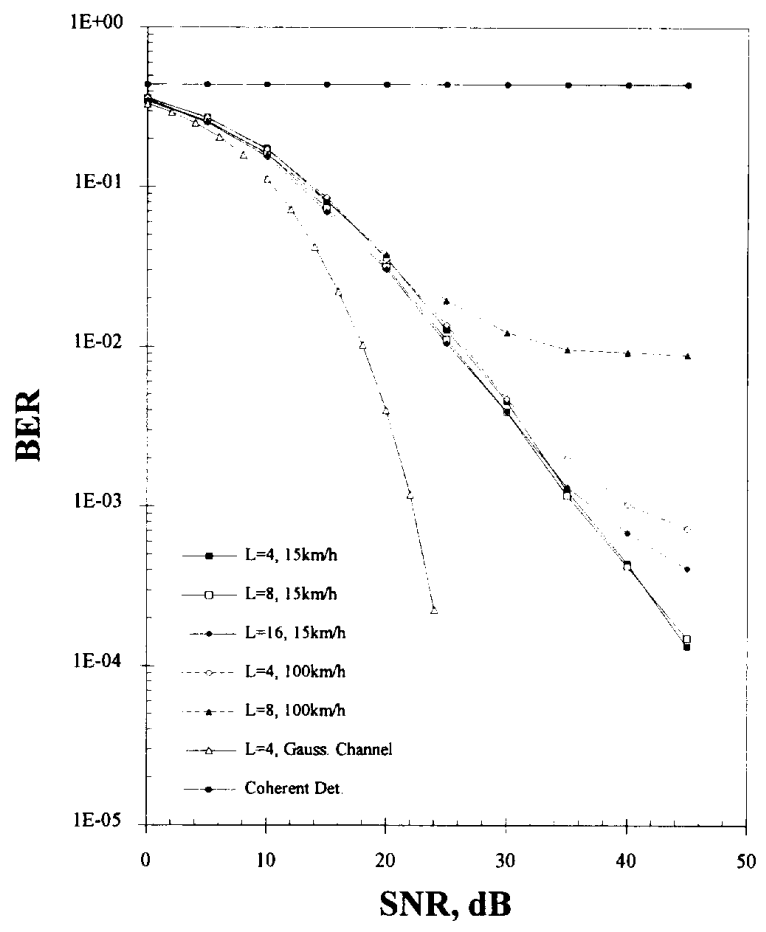

Figure 7 Performance of Pilot Symbol-Aided Square I6QAM in Rayleigh Channels

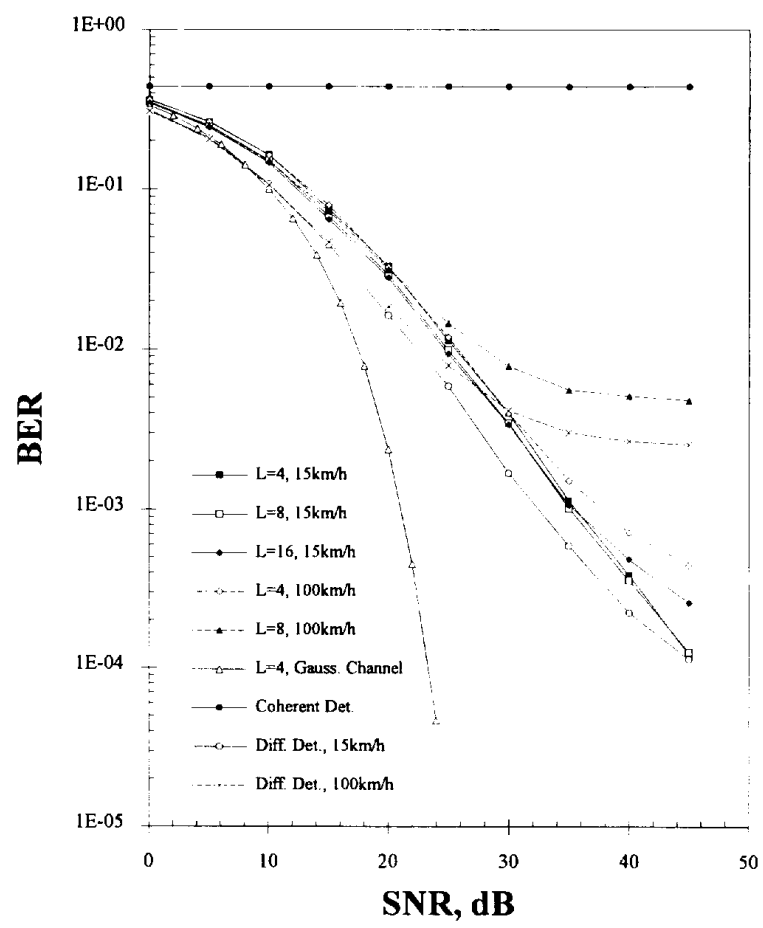

Figure 8 Performance of Pilot Symbol-Aided Star-16QAM in Rayleigh Channels 